\section{Database research}

W ith the onset of medicare, provinces began to amass databases to administer the program (ie, to pay physicians). From the earliest days, some of these databases identified individual patients who received medical services, thus allowing the patients to be 'tracked' through the system, at least as far as physician visits and hospitalizations were concerned. Payment to the physician required a diagnosis as well as a patient identifier. These databases were merged with the province's population database, with Canadian Vital Statistics Offices and, subsequently, with pharmacy databases. The result was a potentially complete description of medical care in an unselected (provincial) population. In most provinces, there are holes in the database, mostly related to services delivered by physicians with special contractual arrangements (ie, no fee-for-service from some family doctors, cancer centres and sometimes emergency rooms); however, these holes are seldom large enough to render the database useless.

The first academics to recognize the scientific potential of provincial medicare databases were those who studied the process of health care and health care utilization. For these people, the database was exactly what they wanted to study, and the more comprehensive and accurate it was, the better. For obvious reasons, provincial Departments of Health were also interested in health care utilization, and they worked with academics to improve their databases and, to the benefit of both parties, make them more accessible. Although the presence and use of the databases posed issues regarding potential invasions of privacy, all parties recognized this and, in Canada, the ethics of database use were effectively worked out relatively early.

Clinical epidemiologists began to utilize Canadian health databases relatively recently (within the past 20 years or so). The advantages for epidemiologists were similar to students of health care utilization; here was a comprehensive record of utilization for a large unselected population, which could be tapped easily and very cheaply. One could do epidemiological studies at one's desk, without going out into the field and sampling and examining a particular population! The problem was that clinical epidemiologists are not usually interested in health care utilization per se, but in what utilization tells them about a particular disease. Thus, if one wants to study drug use or hospitalization, or the relationship between the two, in asthma or chronic obstructive pulmonary disease (COPD) patients, it is important to know whether the people labelled with these diagnoses in the databases actually have them. This is the topic addressed by Lacasse et al (1) in the present issue of the Canadian Respiratory Journal. Studying COPD with the Quebec database, they showed several points of interest. First, the diagnosis of COPD made on the basis of a single physician contact grossly overestimates the prevalence of the disease; in other words, many of these individuals may not have had COPD. Second, in patients older than 40 years of age, the diagnoses of asthma and COPD frequently coexisted, so that studying one disease to the exclusion of the other is probably not possible in this age group. Third, the diagnosis of COPD should be restricted to patients labelled with chronic bronchitis, emphysema or chronic airways obstruction, and should not include other labels.

Lacasse et al (1) recommend that if a database is to be used for epidemiological purposes, its validity should be assessed. This seems reasonable, but it is not easy. One has to establish the presence or absence of a disease in an appropriately sampled population (eg, using spirometry) and then check the population's diagnoses in the database, which is expensive and time consuming. Results depend on the diagnosis and the context of the service. When considering cancer, the validity of databases is excellent. It
Recherche et bases de données

$A$ vec la mise sur pied des régimes d'assuranceAmaladie, les provinces ont commencé à se constituer des bases de données pour administrer leurs programmes (c.-à-d., pour rémunérer les médecins). Dès le début, certaines de ces bases de données identifiaient les patients qui recevaient des services médicaux, ce qui leur permettaient d'être retracés dans le système, du moins en ce qui concernait leurs consultations médicales et leurs hospitalisations puisque la rémunération des médecins reposait sur un diagnostic et un code d'identification des patients. Ces bases de données ont été fusionnées avec les bases de données des populations des provinces, avec le bureau de la statistique de l'état civil du Canada et par la suite, avec les bases de données des pharmacies. Cela a permis de dresser un tableau passablement complet des
soins médicaux prodigués à une population non sélectionnée (provinciale). Et si les bases de données provinciales présentent des lacunes, notamment en ce qui a trait à la prestation de services médicaux en vertu d'arrangements contractuels spéciaux (c.-à-d., autre type de rémunération pour certains médecins de famille, centres d'oncologie et services d'urgence), en revanche, les mailles de ce filet sont rarement assez grandes pour rendre les bases de données totalement inutilisables.

Les premiers chercheurs à avoir reconnu le potentiel scientifique des bases de données des régimes provinciaux d'assurancemaladie sont ceux qui ont étudié le fonctionnement des systèmes de soins de santé et l'utilisation des ressources. Pour ces chercheurs, la base de données était l'objet de leurs travaux et plus elle était complète et précise, plus elle leur était utile. Pour des raisons évidentes, les ministères provinciaux de la santé s'y intéressaient aussi et ils ont ainsi travaillé avec les chercheurs dans le but de raffiner les bases de données et de les rendre plus accessibles, pour le bénéfice des deux parties. Bien que l'existence même des bases de données et leur utilisation aux fins de la recherche aient soulevé quelques questions d'ordre déontologique, protection des renseignements personnels oblige, les parties ont convenu d'y voir, et au Canada, l'éthique a prévalu assez tôt au cours du processus d'utilisation des bases de données.

L'utilisation des bases de données du système de santé canadien par les épidémiologistes cliniques est relativement récente, puisqu'elle a débuté il y a environ 20 ans. Les avantages étaient les mêmes pour les épidémiologistes que pour ceux qui faisaient de la recherche sur l'utilisation des ressources en santé. Ils avaient à leur disposition un dossier global de l'utilisation des ressources par une vaste population non sélectionnée et ils pouvaient facilement le consulter pour ainsi dire sans frais. Les chercheurs pouvaient procéder à une étude épidémiologique dans le confort de leur bureau, sans avoir à se rendre sur le terrain ni à sélectionner des échantillons de population pour les analyser! Le problème, c'est qu'en général, les épidémiologistes cliniques ne s'intéressent pas tant à l'utilisation des ressources en soi, mais bien à ce que celle-ci nous révèle au sujet d'une maladie en particulier. Ainsi, pour quiconque souhaite étudier le recours à tel ou tel médicament ou le taux d'hospitalisation, ou les liens entre les deux, chez des patients atteints d'asthme ou de MPOC, il est important de s'assurer que les diagnostics inscrits dans les bases de données sont justes. C'est ce dont nous parlent Lacasse et coll. (1) dans le présent numéro du Journal canadien de pneumologie. Étudiant la MPOC au moyen de la base de données du Québec, les auteurs ont soulevés plusieurs points intéressants. Tout d'abord, lorsque le diagnostic de MPOC est posé sur la base d'une seule consultation, on obtient une grossière surestimation de la prévalence de la maladie. En d'autres termes, bon nombre de ces sujets ne souffrent peut-être pas de MPOC. Ensuite, chez les patients de plus de 40 ans, les diagnostics d'asthme et de MPOC se chevauchent souvent, de 
is also good when examining a dramatic disease such as myocardial infarction (unpublished observations). It is relatively specific but not sensitive when hospital discharge data are used to identify patients (2). In chronic diseases identified by means of physician claims for office visits, validity is even worse (ie, sensitivity is high, but specificity is low). The agreement between a database and objective evidence is at best 'fair' when young adult asthmatics are studied in this way (3). Perhaps surprisingly, the same level of agreement occurs in diseases like hypertension and diabetes (3).

Is it hopeless to try studying chronic lung diseases using databases? Of course not; the controversy regarding whether inhaled corticosteroids reduce mortality in patients with COPD is based on database research $(4,5)$. Suissa (5) has mined the Saskatchewan database with great profit, making the case that short-acting beta-agonists may impose a serious risk in asthmatic patients. There is some hope even in the data of Lacasse et al (1). It was clear that when the criterion was single physician contact for the 'disease', many people who did not have COPD were labelled with this diagnosis. On the other hand, when multiple visits or drug therapy were the identifying criteria, the patient numbers were credible (based on the expected prevalence of COPD). It seems likely that many of these people did, in fact, have COPD. Given these more stringent criteria, whether one can gain knowledge by using the database depends on the questions asked, but I am on record as believing that inferences regarding COPD management can be drawn from such data (6).

Nick R Anthonisen MD Editor-in-Chief, Canadian Respiratory Journal

\section{REFERENCES}

1. Lacasse Y, Montori VM, Lanthier C, Maltais F. The validity of diagnosing chronic obstructive pulmonary disease from a large administrative database. Can Respir J 2005;12:251-6.

2. Rawson NS, Malcolm E. Validity of the recording of ischaemic heart disease and chronic obstructive pulmonary disease in the Saskatchewan health care datafiles. Stat Med 1995;14:2627-43.

3. Huzel L, Roos LL, Anthonisen NR, Manfreda J. Diagnosing asthma: The fit between survey and administrative database. Can Respir J 2002;9:407-12.

4. Sin DD, Tu JV. Inhaled corticosteroids and the risk of mortality and readmission in elderly patients with chronic obstructive pulmonary disease. Am J Respir Crit Care Med 2001;164:580-4.

5. Suissa S. Effectiveness of inhaled corticosteroids in chronic obstructive pulmonary disease: Immortal time bias in observational studies. Am J Respir Crit Care Med 2003;168:49-53.

6. Anthonisen NR, Woodlrage K, Manfreda J. Use of spirometry and respiratory drugs in Manitobans over 35 years of age with obstructive lung diseases. Can Respir J 2005;12:69-74. sorte que dans cette tranche d'âge, les deux maladies sont pour ainsi dire indissociables. Troisièmement, le diagnostic de MPOC doit être restreint aux patients à qui on ne peut accoler aucune autre étiquette que celles de bronchite chronique, d'emphysème ou d'obstruction des voies respiratoires.

Selon Lacasse et coll. (1), si une base de données doit être utilisée à des fins épidémiologiques, sa validité doit d'abord être confirmée. Cela semble logique, mais c'est plus compliqué qu'il n'y paraît. On doit déterminer la présence ou l'absence d'une maladie auprès d'un échantillon de population approprié (p. ex., à l'aide de la spirométrie), puis, vérifier les diagnostics de la population dans la base de données, ce qui est coûteux et très long. Et les résultats dépendent souvent du diagnostic et du contexte dans lequel les services sont prodigués. Si l'on prend par exemple le cancer, la validité des bases de données est excellente, ce qui vaut également pour les phénomènes aigus, comme l'infarctus du myocarde (observations non publiées). Par ailleurs, les bases de données sont relative ment spécifiques, mais non sensibles lorsqu'on utilise les données sur les congés hospitaliers pour identifier les patients (2). Dans le cas des maladies chroniques recensées par le biais de la facturation des consultations médicales, la validité laisse encore plus à désirer (c.-à-d., la sensibilité est élevée, mais la spécificité est faible). La concordance entre la base de données et la preuve objective est au mieux « acceptable » lorsque c'est une population de jeunes adultes asthmatiques qui est étudiée de cette façon (3). On sera peut-être étonné d'apprendre que le même degré de concor dance s'observe avec des maladies comme l'hypertension et le diabète (3).

Faut-il pour autant renoncer à étudier les maladies pulmonaires chroniques à l'aide de bases de données? Bien sûr que non. La controverse entourant la capacité des corticostéroïdes à réduire la mortalité chez les patients atteints de MPOC trouve son origine dans ce type de recherche $(4,5)$. Suissa $(5)$ a pour sa part interrogé la base de données de la Saskatchewan avec grand succès, affirmant que les bêta-agonistes à action brève étaient associés à un risque grave chez les patients asthmatiques. Même les conclusions de Lacasse et coll. nous permettent d'entretenir un certain espoir (1). Il est vite apparu clair que le diagnostic de MPOC était erroné lorsque le critère d'identification était une seule consultation médicale. Par contre, lorsque plusieurs consultations ou un traitement médicamenteux servaient de critères d'identification, le nombre de cas devenait plus crédible (sur la base de la prévalence de MPOC escomptée). En réalité, bon nombre de ces gens souffraient probablement de MPOC. Ainsi, et même en tenant compte de critères plus stricts, la qualité des renseignements tirées des bases de données dépend toujours des questions qui sont posées au départ, mais à mon avis, les bases de données peuvent mettre au jour certaines inférences susceptibles de faire avancer le traitement de la MPOC (6).

Nick R. Anthonisen, M.D.

Rédacteur en chef, Journal canadien de pneumologie

\section{RÉFÉRENCES}

1. Lacasse Y, Montori VM, Lanthier C, Maltais F. The validity of diagnosing chronic obstructive pulmonary disease from a large administrative database. Can Respir J 2005;12:251-6.

2. Rawson NS, Malcolm E. Validity of the recording of ischaemic heart disease and chronic obstructive pulmonary disease in the Saskatchewan health care datafiles. Stat Med 1995;14:2627-43.

3. Huzel L, Roos LL, Anthonisen NR, Manfreda J. Diagnosing asthma: The fit between survey and administrative database. Can Respir J 2002;9:407-12.

4. Sin DD, Tu JV. Inhaled corticosteroids and the risk of mortality and readmission in elderly patients with chronic obstructive pulmonary disease. Am J Respir Crit Care Med 2001;164:580-4.

5. Suissa S. Effectiveness of inhaled corticosteroids in chronic obstructive pulmonary disease: Immortal time bias in observational studies. Am J Respir Crit Care Med 2003;168:49-53.

6. Anthonisen NR, Woodlrage K, Manfreda J. Use of spirometry and respiratory drugs in Manitobans over 35 years of age with obstructive lung diseases. Can Respir J 2005;12:69-74. 


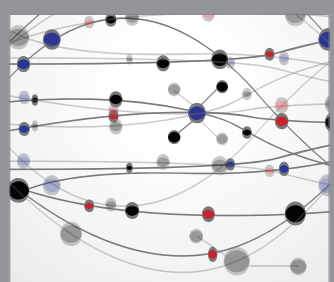

The Scientific World Journal
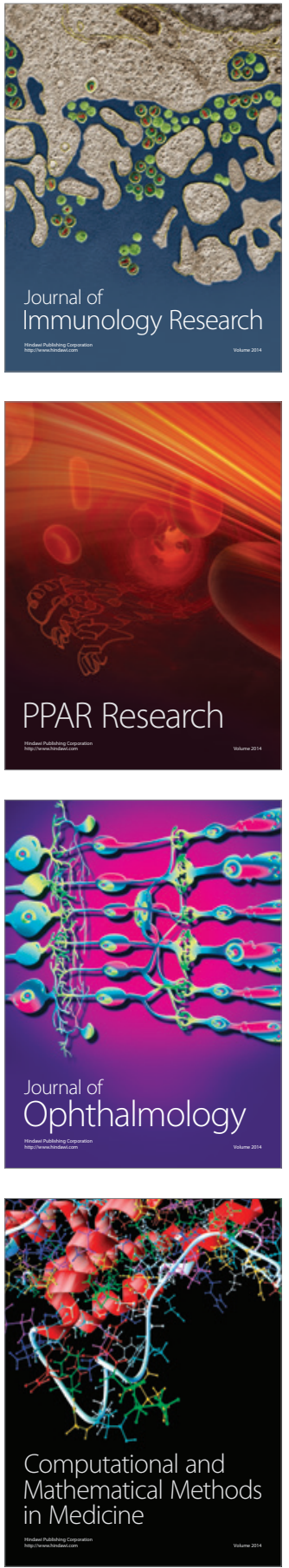

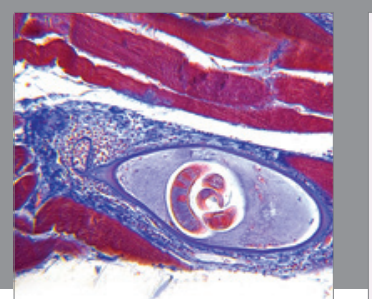

Gastroenterology Research and Practice

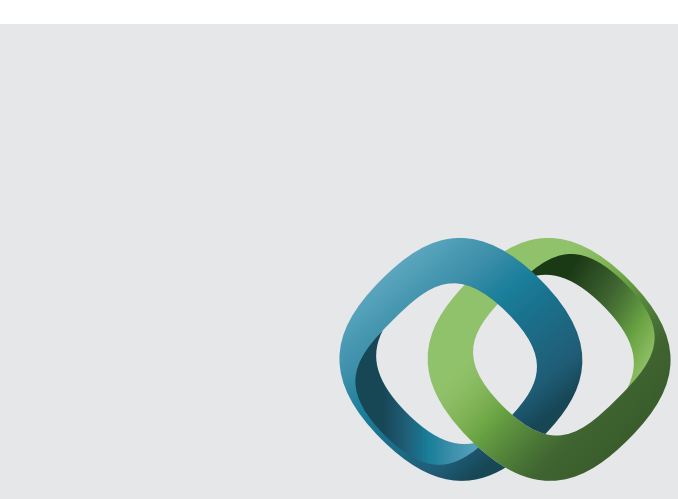

\section{Hindawi}

Submit your manuscripts at

http://www.hindawi.com
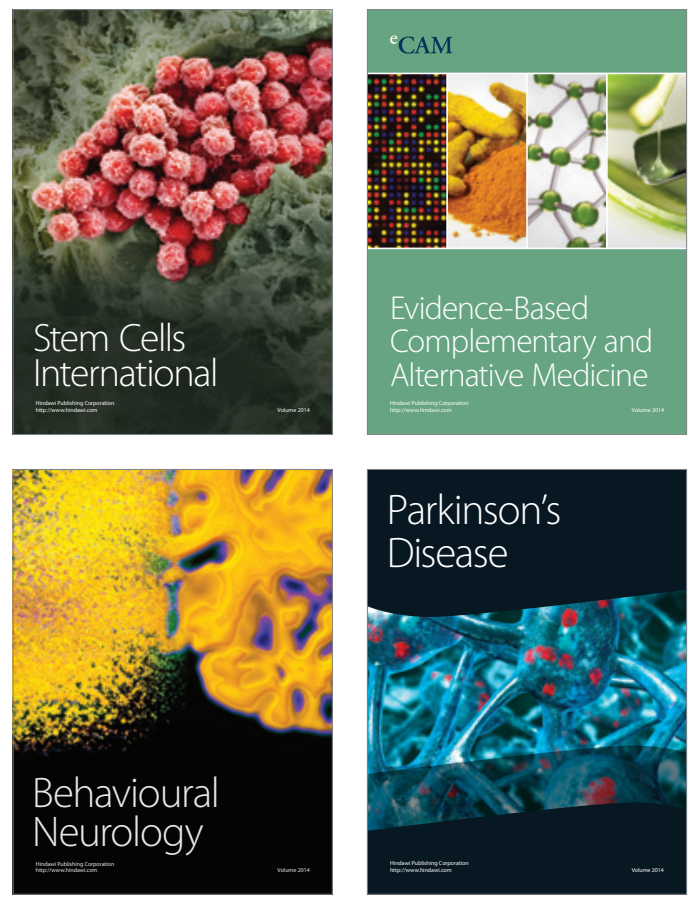
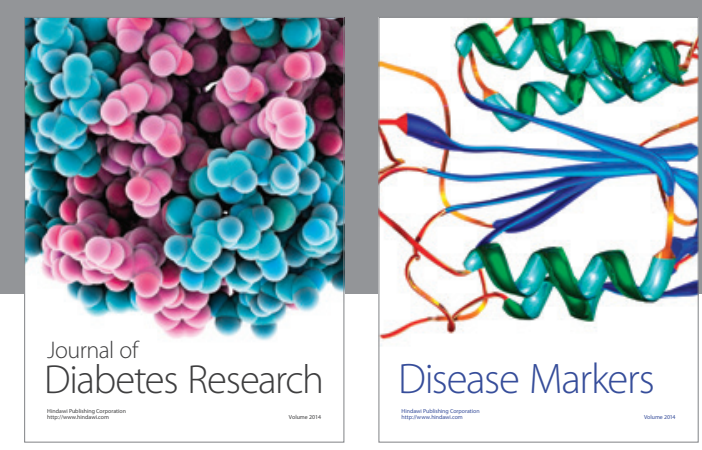

Disease Markers
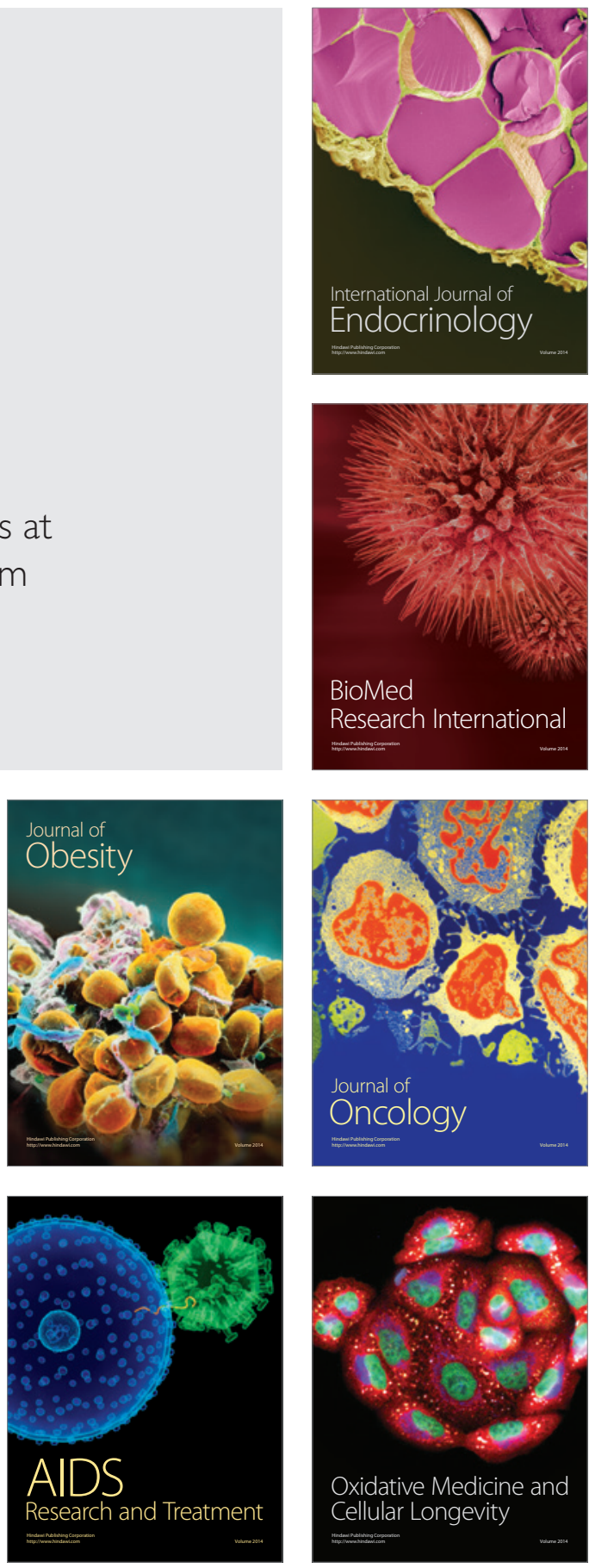